\title{
Cerebellar cavernoma
}

\author{
Authors: Shanzay Bukhari, Asma Naeem and Rajeev Upreti
}

\section{Introduction}

A cavernoma is a cluster of abnormal blood vessels, usually found in the brain and spinal cord. They're sometimes known as cavernous angiomas, cavernous haemangiomas or cerebral cavernous malformation. A case report of a young male with cavernomas is reported.

\section{Methods}

A 37-year-old man was referred to the ambulatory care unit from the accident and emergency department with a 2-day history of visual disturbance and dizziness. Clinical examinations of all systems were normal including neurologic examination which did not reveal any focal neurological signs. Routine haematology and biochemistry blood tests were all normal. Initially, a computed tomography of the brain was requested which showed no definite acute abnormality, but a small focus of high attenuation present within the left middle cerebellar peduncle may represent a small cavernoma. Further assessment with magnetic resonance imaging was suggested. The patient was then admitted to the acute medical unit for further investigations and workup.

\section{Results and discussion}

An MRI of the brain was performed and revealed multifocal cerebral microhaemorrhages of variable sizes spread randomly throughout the supra- and infra-tentorial compartments of the brain with no predilection for the deep structures (basal ganglia/thalami) to suggest chronic hypertensive encephalopathy. Cavernoma are less common in males at this age, which makes our case more unique.

\section{Conclusion}

A detailed history (personal and family) are key to detect such malformations in young patients, which also helps physicians be aware of its related complexities and further management.

\section{Conflict of interest statement}

None declared. 